\title{
Fully Consistent Finite-Strain Landau Theory for High-Pressure Phase Transitions
}

\author{
A. Tröster* \\ Vienna University of Technology, Wiedner Hauptstrasse 8-10/136, A-1040 Vienna, Austria \\ W. Schranz \\ University of Vienna, Boltzmanngasse 5, A-1090 Vienna, Austria
}

F. Karsai and P. Blaha

Vienna University of Technology, Getreidemarkt 9/165-TC, A-1060 Vienna, Austria

(Received 29 August 2013; revised manuscript received 11 February 2014; published 17 July 2014)

Landau theory (LT) is an indispensable cornerstone in the thermodynamic description of phase transitions. As with structural transitions, most applications require one to consistently take into account the role of strain. If temperature drives the transition, the relevant strains are, as a rule, small enough to be treated as infinitesimal, and therefore one can get away with linearized elasticity theory. However, for transitions driven by high pressure, strains may become so large that it is absolutely mandatory to treat them as finite and deal with the nonlinear nature of the accompanying elastic energy. In this paper, we explain how to set up and apply what is, in fact, the only possible consistent Landau theory of high-pressure phase transitions that systematically allows us to take these geometrical and physical nonlinearities into account. We also show how to incorporate available information on the pressure dependence of elastic constants taken from experiment or simulation. We apply our new theory to the example of the high-pressure cubic-tetragonal phase transition in strontium titanate, a model perovskite that has played a central role in the development of the theory of structural phase transitions. Armed with pressure-dependent elastic constants calculated by density-functional theory, we give an accurate description of recent high-precision experimental data and predict a number of elastic transition anomalies accessible to experiments.

DOI: 10.1103/PhysRevX.4.031010

\section{INTRODUCTION}

High-pressure phase transitions in crystals constitute a central research area of modern physics that attracts widespread interest, ranging from astrophysics and geology to chemistry and nanotechnology. Experimentally, this brisk activity is stimulated by the permanent refinement of diamond-anvil-cell techniques, while the ongoing theoretical and hardware-related advances also allow quite precise $a b$ initio calculations of high-pressure transitions. In comparison, even nowadays, the sophistication of the theoretical concepts that are employed to analyze and interpret the high-quality data produced by these methods still leaves a lot to be desired.

The reason for this deplorable situation is not hard to see. In condensed-matter physics, the group-theoretic analysis of symmetry changes at phase transitions is a central concept. Combined with thermodynamics, the resulting machinery of irreducible representations, order parameters (OPs), possible domain states, etc., which comes under the

\footnotetext{
*andreas.troester@tuwien.ac.at
}

Published by the American Physical Society under the terms of the Creative Commons Attribution 3.0 License. Further distribution of this work must maintain attribution to the author(s) and the published article's title, journal citation, and DOI.
Subject Areas: Condensed Matter Physics

name of Landau theory, has proven its value countless times as one of the most useful and versatile approaches to gain both a qualitative as well as a quantitative understanding of phase transitions. In the Landau theory (LT) of solid-state structural transitions, strain usually plays an important role as a primary or secondary OP [1]. For temperature-driven structural transitions and/or at small applied external pressures, strain effects may be small enough to allow the involved strain components to be treated as infinitesimal. The elastic energy may then be truncated beyond harmonic order. Consistency then demands that we sacrifice the possibility of a pressure dependence of the "bare" elastic constants $C_{i j}^{0}$ characterizing the high-symmetry phase. However, once the external pressure comes close to the value of the crystal's elastic constants (typically, some $100 \mathrm{GPa}$ ), ignoring this pressure dependence and other nonlinear effects is bound to result in errors that can quickly approach $100 \%$.

Despite these obvious shortcomings, which are discussed in more detail in the Supplemental Material [2], infinitesimal strains continue to be frequently employed in the Landau analysis of high-pressure transition data [3-7]. Trying to circumvent the technical and conceptual difficulties of nonlinear elasticity theory, many authors are tempted to resort to approximations that are difficult to justify. Bold manipulations like fitting volume data to a 
nonlinear equation of state while continuing to treat strains as infinitesimal and introducing pressure-dependent elastic constants in an ad hoc manner inevitably yield unacceptable internal theoretical contradictions. For instance, there is an important tensorial consistency relation between the pressure dependence of the elastic constants and that of the unit-cell parameters [8], which is generously ignored in all bruteforce attempts to introduce an ad hoc $P$ dependence $C_{i j}^{0}=$ $C_{i j}^{0}(P)$ of the bare elastic constants. In passing, we note that it usually remains unclear why other parameters of the Landau potential that are used should still be assumed to be pressure independent. Even more importantly, nonlinear elasticity theory carefully distinguishes between second derivatives of the free energy taken with respect to various strain measures, and the resulting "elastic constants," which are known as, e.g., Birch, Voigt, Fuchs, and Huang coefficients $[9,10]$, differ from each other by terms of order $P$. Erroneous use of these quantities may thus easily introduce dramatic errors into the analysis of high-pressure data. (See the discussion in Ref. [11].) Such misconceptions are particularly disastrous to eigenvalue calculations, like in applications of the Born stability criteria. (For a recent example, see Ref. [7].) The purpose of this paper is to present the general construction of a LT coupled to finite strains, thereby resolving all these inaccuracies and inconsistencies. The practical value of our new theory is illustrated in an application to recent high-precision measurements of the 10-GPa phase transition in strontium titanate (STO).

\section{LANDAU THEORY COUPLED TO NONLINEAR ELASTICITY}

Mathematically, the assumption of infinitesimally small strain yields dramatic simplifications in the general theory of elasticity, and the resulting theoretical framework of linearized elasticity has found countless applications [12]. However, in the context of structural phase transitions, these assumptions may not always be justified. Important ambient-pressure examples in which the relevant strains are so large that it is mandatory to introduce a proper finitestrain measure such as the Lagrangian strain tensor and eventually even deal with elastic anharmonicity are, e.g., martensitic phase transformations [13], twinning at strongly first-order phase transformations [14], and reconstructive phase transformations [15]. In the context of highpressure phase transitions, the question of how to construct a corresponding Landau theory coupled to finite strain has been addressed for the first time in Ref. [16]. The central idea of this approach was that even if the total observed strain as measured by a Lagrangian strain tensor $\underline{\eta}$ is far from being infinitesimal, its actual spontaneous contribution $\hat{\epsilon}$ originating from the emergence of a nonzeroequilibrium value $\overline{\boldsymbol{Q}}=\overline{\boldsymbol{Q}}(P)$ of the OP $\boldsymbol{Q}$ is still bound to be "small" near a second-order or weakly first-order phase transition. To separate $\hat{\epsilon}$ from $\underline{\eta}$, three reference systems connected by the scheme

$$
\boldsymbol{X} \underset{\underline{\alpha}, \underline{\eta}}{\stackrel{\underline{a}, \underline{e}}{\longrightarrow} \widehat{\boldsymbol{X}} \stackrel{\widehat{\widehat{\alpha}}, \widehat{\underline{\epsilon}}}{\longrightarrow}} \widehat{\widehat{\boldsymbol{X}}}
$$

were introduced in Ref. [16]: (i) the fully deformed system $\hat{\hat{X}}$ (denoted as $\hat{\boldsymbol{Y}}$ in Ref. [16]) at pressure $P$ and relaxedequilibrium value $\overline{\boldsymbol{Q}}=\overline{\boldsymbol{Q}}(P)$; (ii) the undeformed zeropressure "laboratory" or "ambient system" or "frame" denoted by $\boldsymbol{X}$, relative to which the state $\hat{\hat{\boldsymbol{X}}}$ corresponds to the experimentally measured deformation tensor $\underline{\alpha}$ with components $\alpha_{i j}=\partial \hat{\hat{X}}_{i} / \partial X_{j}$ and the resulting Lagrangian strain $\underline{\eta}=(1 / 2)\left(\underline{\alpha}^{T} \cdot \underline{\alpha}-\mathbb{1}\right)$; (iii) a "background reference system" $\hat{X}$ defined as the (hypothetical) state of the system at pressure $P$ and clamped OP $\boldsymbol{Q} \equiv 0$. Relative to $\hat{\boldsymbol{X}}$, one would thus precisely measure the spontaneous strain $\hat{\epsilon}=$ $(1 / 2)\left(\hat{\alpha}^{T} \cdot \hat{\alpha}-\mathbb{1}\right)$ accompanying a deformation gradient matrix $\hat{\alpha}$. $\hat{\boldsymbol{X}}$ and $\boldsymbol{X}$ are related through a deformation gradient tensor with components $a_{i j}=\partial \hat{X}_{i} / \partial X_{j}$ and the resulting Lagrangian strain tensor with components $e_{i j}=$ $(1 / 2)\left(a_{k i} a_{k j}-\delta_{i j}\right)$ as $\underline{e}=(1 / 2)\left(\underline{a}^{T} \cdot \underline{a}-\mathbb{1}\right)$. Given these definitions, the total experimentally observed strain is thus decomposed into the nonlinear [17] superposition

$$
\eta_{i j}=e_{i j}+a_{k i} \hat{\epsilon}_{k l} a_{l j}
$$

In the background reference frame $\hat{\boldsymbol{X}}$, the $\boldsymbol{Q}$-independent elastic contribution $F_{0}(\hat{\epsilon} ; \hat{\boldsymbol{X}})$ to the Landau free energy is assumed to be captured by the harmonic approximation

$$
\frac{F_{0}(\hat{\epsilon} ; \hat{\boldsymbol{X}})}{V[\hat{\boldsymbol{X}}]} \approx \sum_{i j} \tau_{i j} \hat{\epsilon}_{i j}+\frac{1}{2} \sum_{i j k l} C_{i j k l}[\hat{\boldsymbol{X}}] \hat{\epsilon}_{i j} \hat{\epsilon}_{k l}
$$

involving the external stress tensor $\tau_{i j}$ and the elastic constants $C_{i j k l}[\hat{\boldsymbol{X}}]$ of the background system. Furthermore, in accordance with the traditional approach of LT, the fact that both OPs as well as strain components remain small in the vicinity of the transition suggests that we drop all coupling terms between strain and OP beyond second order in $\boldsymbol{Q}$. For simplicity, we take the OP to be scalar and content ourselves with a single coupling between $Q$ and $\hat{\epsilon}$ of type $\sum_{i j} Q^{2} d_{i j}(\hat{\boldsymbol{X}}) \hat{\epsilon}_{i j}$, which yields the ansatz

$$
\begin{aligned}
\frac{F(Q, \hat{\epsilon} ; \hat{\boldsymbol{X}})}{V(\hat{\boldsymbol{X}})}= & \Phi(Q ; \hat{\boldsymbol{X}})+Q^{2} \sum_{i j} d_{i j}(\hat{\boldsymbol{X}}) \hat{\epsilon}_{i j} \\
& +\frac{F_{0}(\hat{\epsilon} ; \hat{\boldsymbol{X}})}{V[\hat{\boldsymbol{X}}]},
\end{aligned}
$$

in which we introduce the potential density $\Phi(Q ; \hat{\boldsymbol{X}})$ of the pure OP contribution. In what follows, we shall assume $\Phi(Q ; \hat{\boldsymbol{X}}) \equiv(A[\hat{\boldsymbol{X}}] / 2) Q^{2}+(B[\hat{\boldsymbol{X}}] / 4) Q^{4}+(C[\hat{\boldsymbol{X}}] / 6) Q^{6}$ to be a simple sixth-order polynomial in $Q$. Unfortunately, even 
with these assumptions, the resulting strain-equilibrium conditions assume the highly nonlinear form [16]

$$
\sum_{m n} \frac{\overline{\hat{\alpha}}_{m i} \overline{\hat{\alpha}}_{n j}}{J[\hat{\alpha}]}\left[\bar{Q}^{2} d_{m n}(\hat{\boldsymbol{X}})+C_{m n k l}[\hat{\boldsymbol{X}}] \hat{\epsilon}_{k l}\right] \approx 0,
$$

where $J(\hat{\alpha})=V[\hat{\hat{\boldsymbol{X}}}] / V[\hat{\boldsymbol{X}}]=\operatorname{det} \hat{\alpha}$. To overcome this problem, in Ref. [16], the observation that sufficiently close to the transition the spontaneous strain components are always numerically small was exploited by regarding the spontaneous strain $\hat{\epsilon}^{2}$ as infinitesimal, and the nonlinear prefactors $\overline{\hat{\alpha}}_{m i} \overline{\hat{\alpha}}_{n j} J^{-1}[\overline{\hat{\alpha}}]$ were consequently put to 1 . One then arrives at a system of linear equations that must be solved for the strain components $\hat{\epsilon}_{k l}$ as functions of $\bar{Q}$ by inverting the tensor $C_{m n k l}[\hat{\boldsymbol{X}}]$. However, this step is somewhat delicate because the required invertibility is not automatically guaranteed. Indeed, we have recently realized that one can do a lot better.

In fact, despite its expected numerical smallness, let us continue to regard the spontaneous strain $\overline{\hat{\epsilon}}$ as a full Lagrangian strain tensor. Using the first-order approximations [18] $\overline{\hat{\alpha}}_{i j}=\delta_{i j}+\overline{\hat{\epsilon}}_{i j}+O\left(\overline{\hat{\epsilon}}^{2}\right)$ and $J[\overline{\hat{\alpha}}]=1+\sum_{k} \overline{\hat{\epsilon}}_{k k}+$ $O\left(\overline{\hat{\epsilon}}^{2}\right)$, we expand the geometrical prefactor in Eq. (5) up to harmonic order in $\overline{\hat{\epsilon}}$. A short calculation results in

$$
\bar{Q}^{2} d_{i j}[\hat{\boldsymbol{X}}]+\sum_{k l} B_{i j k l}[\hat{\boldsymbol{X}}] \overline{\hat{\epsilon}}_{k l} \equiv 0,
$$

in which the well-known Birch coefficients $B_{i j k l}[\hat{\boldsymbol{X}}]=$ $C_{i j k l}[\hat{\boldsymbol{X}}]+(1 / 2)\left(\tau_{j k} \delta_{i l}+\tau_{i k} \delta_{j l}+\tau_{j l} \delta_{i k}+\tau_{i l} \delta_{j k}-2 \tau_{i j} \delta_{k l}\right)$ of the background system $\hat{X}$ have taken over the role formerly played by the elastic constants $[17,18]$. Since the background system $\hat{\boldsymbol{X}}$ is defined by the constraint $\bar{Q} \equiv 0$, which inhibits the transition under investigation, application of the Born stability criteria [9,18-22] now ensures that its tensorial inverse

$$
\sum_{m n} B_{i j m n}[\hat{\boldsymbol{X}}] S_{m n k l}[\hat{\boldsymbol{X}}]=\frac{1}{2}\left(\delta_{i k} \delta_{j l}+\delta_{i l} \delta_{j k}\right),
$$

the tensor $S_{i j k l}[\hat{\boldsymbol{X}}]$ of elastic compliances, exists. Inserting the solution of Eq. (6)

$$
\overline{\hat{\epsilon}}_{m n}=-\bar{Q}^{2} \sum_{i j} d_{i j}(\hat{\boldsymbol{X}}) S_{m n i j}[\hat{\boldsymbol{X}}]
$$

into the second equilibrium condition

$$
\Phi^{\prime}(\bar{Q} ; \hat{\boldsymbol{X}})+2 \bar{Q} \sum_{i j} d_{i j}(\hat{\boldsymbol{X}}) \overline{\hat{\epsilon}}_{i j} \equiv 0
$$

and reintegrating by $\bar{Q}$ finally yields (up to an unimportant constant) the renormalized background pure OP potential density

$$
\Phi_{R}(Q ; \hat{\boldsymbol{X}})=\frac{A_{R}[\hat{\boldsymbol{X}}]}{2} Q^{2}+\frac{B_{R}[\hat{\boldsymbol{X}}]}{4} Q^{4}+\frac{C_{R}[\hat{\boldsymbol{X}}]}{6} Q^{6}
$$

with quartic coefficient

$$
B_{R}[\hat{\boldsymbol{X}}]=B[\hat{\boldsymbol{X}}]-2 \sum_{i j k l} d_{i j}[\hat{\boldsymbol{X}}] S_{i j k l}[\hat{\boldsymbol{X}}] d_{k l}[\hat{\boldsymbol{X}}]
$$

while we still maintain $A_{R}[\hat{\boldsymbol{X}}]=A[\hat{\boldsymbol{X}}]$ and $C_{R}[\hat{\boldsymbol{X}}]=C[\hat{\boldsymbol{X}}]$. The equilibrium condition for the OP then takes the simple form

$$
\Phi_{R}{ }^{\prime}(\bar{Q} ; \hat{\boldsymbol{X}}) \equiv 0
$$

All of these quantities are defined with respect to the reference state $\hat{\boldsymbol{X}}=\hat{\boldsymbol{X}}[P]$. It remains to determine this implicit $P$ dependence. As to the elastic constants $C_{i j k l}[\hat{\boldsymbol{X}}]$, this information is, in principle, accessible by density-functional-theory (DFT) calculations. The key observation that allows us to assess the remaining implicit $P$ dependence of the coefficients $A[\hat{\boldsymbol{X}}], B[\hat{\boldsymbol{X}}], C[\hat{\boldsymbol{X}}]$, and $d_{i j}[\hat{\boldsymbol{X}}]$ is the following. Working in the laboratory system $\boldsymbol{X}$, one would be forced to go to prohibitively high powers in the Landau expansion

$$
\begin{aligned}
\frac{F(Q, \underline{\eta} ; \boldsymbol{X})}{V(\boldsymbol{X})} \equiv & \frac{A}{2} Q^{2}+\frac{B}{4} Q^{4}+\frac{C}{6} Q^{2}+\cdots \\
& +\frac{1}{2 !} \sum_{i j k l} C_{i j k l}^{(2)} \eta_{i j} \eta_{k l} \\
& +\frac{1}{3 !} \sum_{i j k l m n} C_{i j k l m n}^{(3)} \eta_{i j} \eta_{k l} \eta_{m n}+\cdots \\
& +\sum_{N=1}^{\infty} Q^{2 N} \sum_{i j} \eta_{i j}\left(d_{i j}^{(2 N, 1)}+\sum_{k l} \frac{d_{i j k l}^{(2 N, 2)}}{2 !} \eta_{k l}\right. \\
& \left.+\sum_{k l m n} \frac{d_{i j k l m n}^{(2 N, 3)}}{3 !} \eta_{k l} \eta_{m n}+\cdots\right)
\end{aligned}
$$

to capture nonlinear elastic effects with sufficient precision. Nevertheless, by definition, all its coefficients are strain-independent (but possibly $T$-dependent) constants. We now compare common coefficients for the monomials $Q^{2 N} \hat{\epsilon}_{i_{1} j_{1}}, \ldots, \hat{\epsilon}_{i_{n} j_{n}}$ in a combined expansion of the invariance relation

$$
F(Q, \hat{\epsilon} ; \hat{\boldsymbol{X}}) \equiv F\left(Q, \underline{e}+\underline{a}^{+} \cdot \hat{\epsilon} \cdot \underline{a} ; \boldsymbol{X}\right)
$$

of the free energy under a change of the strain reference frame. Guided by the requirement that the maximum power of $Q$ appearing on both sides should be identical (i.e., 6 in our present model), a straightforward but somewhat 
lengthy calculation, whose details may be found in the Supplemental Material [2], results in the relations

$$
\begin{aligned}
& J(\underline{a}) d_{s t}[\hat{\boldsymbol{X}}]=\sum_{i j} a_{s i} d_{i j}^{(2,1)} a_{t j}+\sum_{i j k l} a_{s i} d_{i j k l}^{(2,2)} a_{t j} e_{k l}+\cdots \\
& J(\underline{a}) A(\hat{\boldsymbol{X}})=A+2 \sum_{i j} d_{i j}^{(2,1)} e_{i j}+\sum_{i j k l} d_{i j k l}^{(2,2)} e_{i j} e_{k l}+\cdots \\
& J(\underline{a}) B(\hat{\boldsymbol{X}})=B, \quad J(\underline{a}) C(\hat{\boldsymbol{X}})=C .
\end{aligned}
$$

To judge the usability of these power series in the background strains $e_{k l}$, assume that the numerical values for the coefficients $A, B, C, d^{(2,1)}$, and $C_{i j k l}^{(2)}$ defining the zeropressure theory are known, as is the case in many applications. In turn, the requirement that our high-pressure extension of standard ambient-pressure Landau theory reduces to the ambient potential in the low-pressure limit is a fundamental constraint that results in a drastic reduction of the number of unknown parameters of our theory. Beyond knowledge of the ambient-pressure coupling coefficients, the pressure dependence of the background elastic constants $C_{i j k l}^{0}(P) \equiv C_{i j k l}[\boldsymbol{X}(P)]$ is another required "external" input to our theory. In rare cases, experimental data may be available that allow their sufficiently precise extrapolation beyond the critical pressure $P_{c}$ for the specific system under consideration, but otherwise, we will resort to DFT to provide this information. From knowledge of the relevant tensor components $C_{i j k l}^{0}(P)$, we compute the components $e_{i j}(P)$ of the background strain (see Ref. [16]). Of the remaining list of relevant couplings introduced above, only the coefficients $d_{i j \ldots .}^{(2, \alpha)}$, $\alpha=2,3, \ldots$, appearing in Eqs. (15)-(17), which cover the possible nontrivial $P$ dependencies of the quadratic orderparameter coefficient $A[\hat{\boldsymbol{X}}]$ and the couplings $d_{i j}[\hat{\boldsymbol{X}}]$ between order parameter and strain, must be dealt with. Superficially, we may thus still face a large number of unknowns. However, the symmetry of the low-pressure phase can be used to further reduce their number. In fact, assume for simplicity that the low-pressure phase is cubic. Then, $e_{i j} \equiv e \delta_{i j}$ is diagonal, and Eqs. (14) collapse to

$$
\begin{gathered}
a d_{i j}[\hat{\boldsymbol{X}}]=d_{i j}^{(2,1)}+d_{i j}^{(2,2)} e+d_{i j}^{(2,3)} e^{2}+\cdots \\
a^{3} A(\hat{\boldsymbol{X}})=A+2 d_{i i}^{(2,1)} e+d_{i i}^{(2,2)} e^{2}+\frac{d_{i i}^{(2,3)}}{3} e^{3}+\cdots,
\end{gathered}
$$

in which only certain sums

$$
d_{i j}^{(2,2)}:=\sum_{k} d_{i j k k}^{(2,2)}, \quad d_{i j}^{(2,3)}:=\sum_{k l} d_{i j k k l l l}^{(2,3)}, \ldots
$$

of these unknown coefficients remain as parameters. Inspection of Eqs. (15) and (16) reveals this $P$ dependence, which is, however, not given by a fractious Taylor expansion in powers of $P$ but implicitly encoded in terms of a well-tempered power-series expansion of the finite but rather small dimensionless quantities $e_{i j}(P)$, which is an enormous numerical advantage of our present Helmholtz (i.e., strain-parametrized) approach over the more simpleminded Gibbs-type (i.e., pressure-parametrized) attempts based on introducing $P$ dependence into LT by expanding the coefficients into mere powers of $P$. If we keep only the lowest expansion coefficients $d_{i j}^{(2, \alpha)}$ for $\alpha=2,3$, the coefficient $A[\hat{\boldsymbol{X}}]$ is determined up to terms of order $e^{4}(P)$ and $d_{i j}[\hat{\boldsymbol{X}}]$ is fixed up to corrections of order $e^{3}(P)$.

In practice, the resulting parametrization is numerically still somewhat inconvenient. Rather, we would like to treat the value of the critical pressure $P_{c}$ as an input parameter to the fit, since experimentally, this quantity can frequently be assessed with quite good precision by combining different experimental techniques, while it tends to come out less precise in a least-squares fit of pure strain data contaminated by unavoidable errors. Thus, it is usually a good idea to trade one of the fit parameters $d_{i j}^{(2, \alpha)}$ for $P_{c}$ by solving the implicit equation $A\left[\hat{\boldsymbol{X}}\left(P_{c}\right)\right] \equiv 0$.

\section{APPLICATION TO STRONTIUM TITANATE}

We illustrate the advantages of our present approach by performing a fit of recent high-precision measurements [23] of the pressure-induced $P m \overline{3} m \leftrightarrow I 4 / \mathrm{mcm}$ transition around $P_{c}=9.6 \mathrm{GPa}$ in the model-perovskite strontium titanate at room temperature. At ambient pressure, $\mathrm{SrTiO}_{3}$ was already shown in the 1960s to undergo a cubic $\rightarrow$ tetragonal transition at $T_{c} \approx 105 \mathrm{~K}$ recognized to be an archetypal model for other soft-mode-driven structural phase transitions [24]. The crystal class of perovskites itself is also of widespread interest for a number of technological applications [25]. Moreover, recent research [26] indicates that more than $93 \%$ by volume of Earth's lower mantle consists of minerals of the perovskite structure.

A glance at Eq. (1) of Ref. [23] indicates that the ambient-pressure Landau potential underlying the 105-K transition is just of the structure discussed above, and we immediately inherit the whole set of ambient-pressure parameters $A_{0}, B, C, d_{11}^{(2,1)}$, and $d_{33}^{(2,1)}$ (see Ref. [27]) from the left column of Table III of Ref. [23]. To provide the only missing further input for applying our present nonlinear theory, we calculate the background elastic constants $C_{11}^{0}(P)$ and $C_{12}^{0}(P)$ (reverting to Voigt notation) from total energies in DFT, using the WIEN2K package [28]. In addition to using the standard local density approximation for the exchange-correlation functional, calculations were carried out based on the Perdew-Burke-Ernzerhof (PBE) $[29,30]$ and Wu-Cohen [31] approximations as well as on the PBESol [32] functional, a version of PBE optimized for application to solids. While we refer to the Supplemental 
Material for more details on these calculations [2], here, we content ourselves with reporting that the results based on the latter two functionals are found to be practically indistinguishable within the considered pressure range. Moreover, even though the PBE functional shows a slightly better match to the observed pressure dependence of lattice parameters than the PBESol and Wu-Cohen functionals, the PBESol and Wu-Cohen functionals give the best overall results when plugged into our nonlinear LT, which is most probably due to their different predictions of elastic constants. With increasing $P, C_{11}^{0}(P)$ is found to increase roughly linearly from 349.3 to about $895.9 \mathrm{GPa}$, while $C_{12}^{0}(P)$ increases from 103.1 to $208.1 \mathrm{GPa}$ between 0 and $70 \mathrm{GPa}$. (See the dotted lines in Fig. 3 and the more detailed discussion in the Supplemental Material [2].)

As the low-pressure symmetry of STO is cubic, Eq. (17) applies. Following Ref. [23], we also the take the experimental value of $P_{c}=9.6 \mathrm{GPa}$ for the critical pressure as an input parameter to our theory. In view of the arguments presented below Eq. (17), very little freedom for fitting our theory to the available experimental data is left, since only three parameters, say, $d_{11}^{(2,2)}, d_{33}^{(2,2)}$, and $d_{11}^{(2,3)}$, remain.

Despite this constricting corset, a fit based on these remaining three parameters works amazingly well, as is revealed in Fig. 1. The figure's insets also expose the complete failure of the plain infinitesimal approach [23]. Before we discuss the behavior of the LT coupling constant emerging from this fit, let us first examine the corresponding prediction of the static longitudinal equilibrium elastic constants of STO. By a rather straightforward generalizing of the approach of Ref. [33] to the nonlinear regime, whose details are given in the Supplemental Material [2], we can compute their $P$-dependent transition anomalies. The results of these calculations, which are gathered in Fig. 3, reveal that while the anomaly in the bulk modulus is not very dramatic (but cf. Ref. [34]), the anomalies

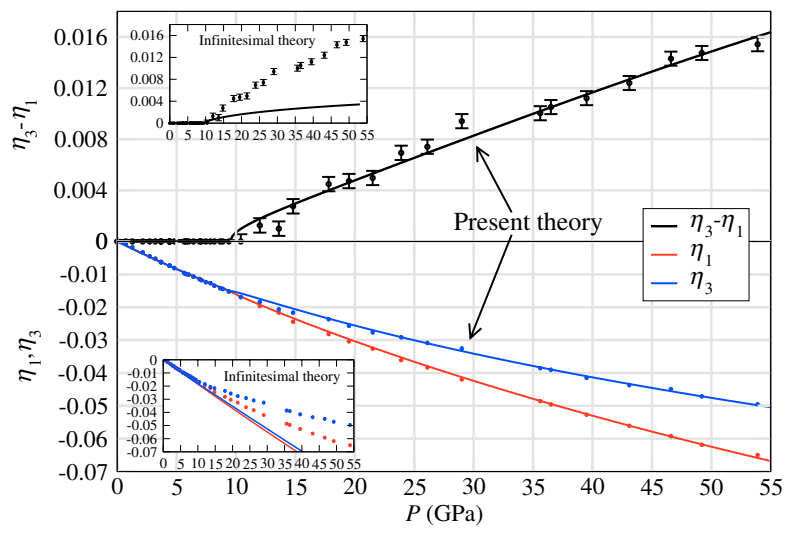

FIG. 1. $\quad P$ dependence of Lagrangian strain components $\eta_{1}(P)$ and $\eta_{3}(P)$ (solid colored lines) as obtained from a fit of our theory to the experimental data of Ref. [23] (black data points). Insets: Corresponding results of the infinitesimal approach using the parameters of the left column of Table III of Ref. [23].

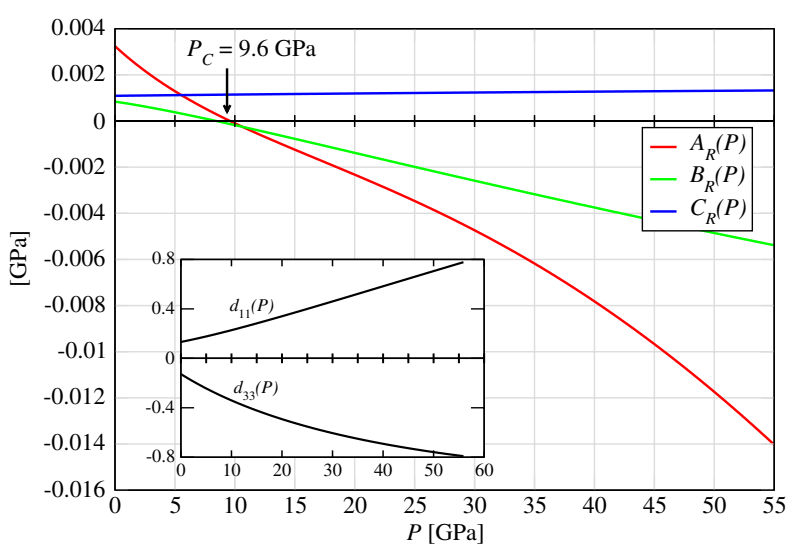

FIG. 2. Effective $P$ dependence of Landau coefficients $A_{R}(P) \equiv A_{R}[\hat{\boldsymbol{X}}], \quad B_{R}(P) \equiv B_{R}[\hat{\boldsymbol{X}}]$, and $C_{R}(P) \equiv C_{R}[\hat{\boldsymbol{X}}]$. Note the change of sign of the coefficient $B_{R}(P)$ near $P=P_{c}$, which implies the tendency of the transition toward becoming tricritical or even weakly first order under pressure. Inset: $P$ dependence of parameters $d_{i i}(P) \equiv d_{i i}[\hat{\boldsymbol{X}}]$.

observed for the individual elastic tensor components are quite pronounced: $C_{11}, C_{12}$, and $C_{33}$ exhibit a notable negative jump at $P=P_{c}$, while $C_{13}$ undergoes an upward jump. While these findings might have been anticipated from a qualitative comparison to the $T$-driven transition [35], they still await their verification in experiment.

We now turn to the discussion of the $P$ dependence of the various coupling constants of our theory that are more difficult to anticipate by someone used to working with the infinitesimal theory. First of all, besides the discussed transition anomalies, Fig. 3 also illustrates the strong $P$ dependence of the background elastic constants that are computed from the DFT simulations and that are qualitatively to be expected for any high-pressure phase-transition scenario. Of course, this fact has enormous implications for the behavior of the remaining couplings in the theory. As to $A_{R}(P) \equiv A_{R}[X(P)]$, Fig. 2 shows that the resulting $P$

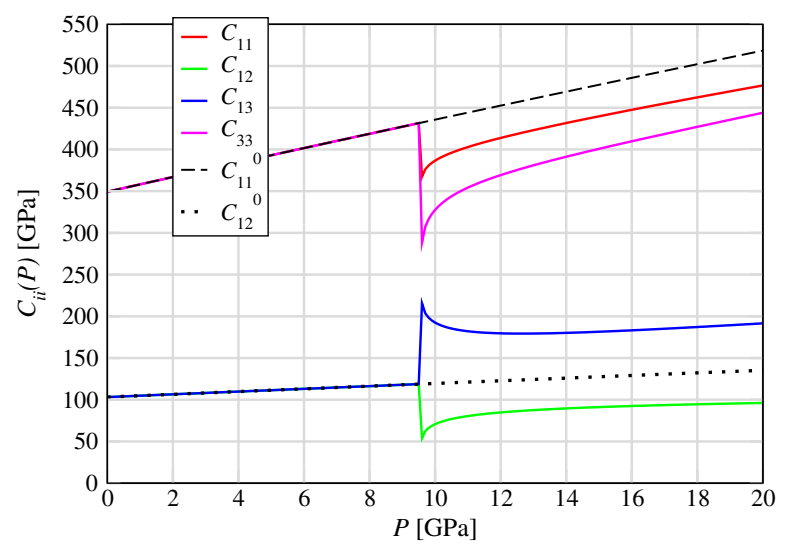

FIG. 3. Solid colored lines: Pressure dependencies of elastic constants $C_{11}(P), C_{12}(P), C_{13}(P)$, and $C_{33}(P)$ as determined from combining our present theory with the approach of Ref. [33]. Dotted black lines: Background elastic constants. 
dependence still does not deviate too far from the linear slope one would expect from standard LT (see the Supplemental Material [2]). However, in sharp contrast to the "usual" assumptions made by practitioners of LT, $B_{R}(P) \equiv B_{R}[\boldsymbol{X}(P)]$ also decreases strongly with increasing $P$ and even crosses 0 near $P_{c}$. From an infinitesimal approach, we could have neither deduced this increasing tendency of the transition to become tricritical or even weakly first order under pressure, as it is implied by this behavior of $B_{R}(P)$. Nor could we have anticipated the considerable but smooth growth in modulus of the couplings $d_{11}(P)$ and $d_{33}(P)$ over the considered pressure range shown in the inset of Fig. 2.

Another rule of thumb verified in hundreds of applications of LT to temperature-driven structural phase transitions, which many researchers usually take for granted in judging experimental data, is that the squared OP and spontaneous strain are proportional for situations characterized by a linear-quadratic coupling between strain and OP. This expectation is rooted in the fact that for the infinitesimal theory, an equation holds that formally resembles our Eq. 7). However, in the present situation, the proportionality factor $-\sum_{i j} d_{i j}(\hat{\boldsymbol{X}}) S_{m n i j}[\hat{\boldsymbol{X}}]$ between $\overline{\hat{\epsilon}}_{m n}$ and $\bar{Q}^{2}$ cannot be taken to be a mere constant like in the infinitesimal case but may strongly depend on $P$. We emphasize that this nontrivial $P$ dependence not only results from that of the background elastic constants but also from the pressure dependence of the coupling coefficients $d_{i j}[\hat{\boldsymbol{X}}]$, which, as the inset of Fig. 2 demonstrates,

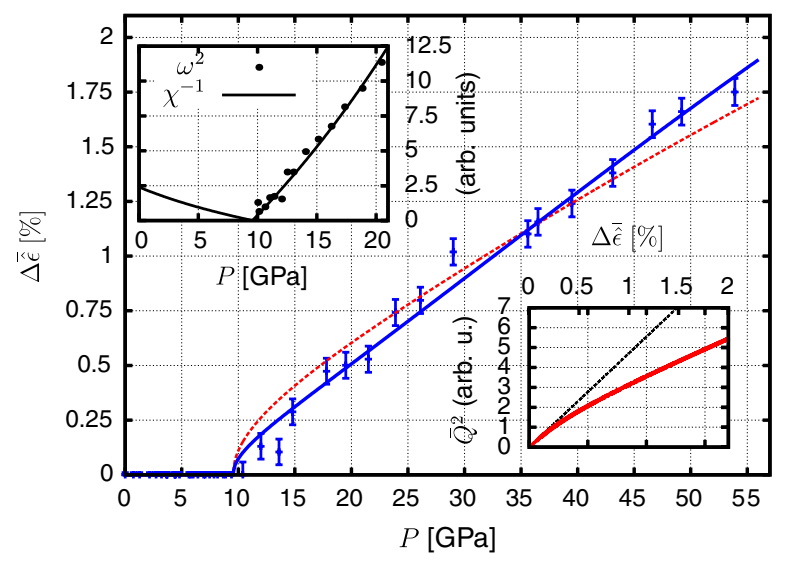

FIG. 4. Main plot: Difference $\Delta \overline{\hat{\epsilon}}(P)=\overline{\hat{\epsilon}}_{3}-\overline{\hat{\epsilon}}_{3}$ of spontaneous strain components as obtained from experiment vs our fit (blue data points and function). For comparison, the dotted red line shows our result for the squared order parameter $\bar{Q}^{2}(P)$ after a suitable rescaling. Left upper inset: Experimental data for the squared soft-mode frequency compared to a suitable rescaled fit result for the inverse order-parameter susceptibility $\chi^{-1}(P)$ as obtained from our fit. Right lower inset: $\Delta \overline{\hat{\epsilon}}$ parametrized as a function of the squared equilibrium order parameter $\bar{Q}^{2}$. The dotted line corresponds to an assumed proportionality between $\Delta \overline{\hat{\epsilon}}(P)$ and $\bar{Q}^{2}(P)$ as it would follow from a naive application of LT. can be quite substantial. For STO, the breakdown of the simple proportionality $\overline{\hat{\epsilon}}_{m n} \propto \bar{Q}^{2}$ for the high-pressure scenario is illustrated in Fig. 4.

In passing, we note that within the standard folklore of LT, it is also quite tempting to identify the squared softmode frequency $\omega^{2}(P)$ with the inverse order-parameter susceptibility $\chi^{-1}(P)$ up to proportionality. However, this identification may also be questionable in the high-pressure setting. Besides the second derivative of the free energy, inertial terms also appear in the dynamical equation governing the soft-mode behavior, and these inertial terms may exhibit an additional pressure dependence. That being said, a fit of $\chi^{-1}(P)$ as deduced from our theory to the experimental data for $\omega^{2}(P)$ taken from Ref. [23] is depicted in the upper left inset of Fig. 4.

The above discussion of the various nontrivial consequences of the delicate but inevitable $P$ dependence of high-pressure LT, which is successfully taken into account in our new theory, should cast severe doubts on any physical prediction that is merely based on a traditional infinitesimal strain approach or simple ad hoc generalizations of it.

\section{SUMMARY AND DISCUSSION}

Our present nonlinear LT offers a complete quantitative description of the experimentally measured strains and their transition anomalies. In fact, it seems very hard to further simplify the theory we have presented without sacrificing mathematical consistency, numerical precision, or both. Let us discuss a few important remaining points while summarizing.

As we have shown above, in building a LT for high pressure, it is essential to replace the infinitesimal strain tensor by a finite-strain measure like the Lagrangian strain tensor. Only then it is possible to disentangle the spontaneous strain, which, by definition, accompanies the appearance of a nonzero order parameter beyond the critical pressure $P_{c}$, from the background strain, which results from the application of external pressure by introducing an elastic background reference system and plays a role similar to other "baselines" introduced in the description of phase-transition anomalies. It is only this spontaneous strain, which is numerically small enough to allow a harmonic approximation of its associated elastic energy. The coupling coefficients of the resulting LT, which are defined with respect to the background reference system, formally exhibit a pressure dependence that can be expanded in a well-behaved power series built from the components of the background strain tensor. For obtaining correct estimates of these pressure dependences from a fit, independent "external" information on the pressure dependence of the elastic constants from DFT or experiment is absolutely essential.

We close with a short technical comment. Even though the determination of the pressure dependence of the LT 
coupling constants seems to be the central issue at the outset, we chose to work in the Helmholtz picture, in which strain rather than stress functions as the external control parameter. This decision was based on the unpleasant fact that whereas stress and strain are thermodynamically conjugate within the approximation of linearized elasticity, such a relation does not hold for the fully nonlinear Lagrangian strain tensor and the Cauchy stress tensor. In fact, the true conjugate of the Lagrangian strain tensor is the so-called Piola-Kirchhoff stress of the second kind, which differs from the physical Cauchy stress tensor by nonlinear correction terms. Technically, this fact implies that it is very cumbersome to introduce a Gibbs potential [17,20,21]. Together with the nonidentity of elastic constants and Birch coefficients, the lack of a simple parametrization in terms of stress is one of the main pitfalls that nonlinear elasticity theory provides for people familiar only with the linearized theory, even though connoisseurs of the classic books of Landau and Lifshitz may already recall a corresponding warning. [See the sentence following Eq. (4.11) of Ref. [12].] On the other hand, our strategy of consistently using strain instead of stress as the control variable is finally rewarded by the tame numerical behavior of the expansions (15) and (16). We would, therefore, advocate the statement that our Helmholtz approach is likely to be the only one that, in practice, leads to a manageable formulation of the theory. Moreover, this strategy is also in complete agreement with the way stress is obtained in standard static DFT calculations. Imposing a certain size and shape of the DFT supercell, it is the strain that is controlled, while stress is only derived a posteriori from, e.g., Hellmann-Feynman arguments.

\section{ACKNOWLEDGMENTS}

A. T. and W. S. acknowledge support by the Austrian Science Fund (FWF) Projects No. P22087-N16 and No. P23982-N20, respectively. F. K. and P. B. acknowledge the TU Vienna doctoral college COMPMAT.

[1] J. Tolédano and P. Tolédano, The Landau Theory of Phase Transitions (World Scientific, Singapore, 1987).

[2] See Supplemental Material at http://link.aps.org/ supplemental/10.1103/PhysRevX.4.031010 for a critical review of the infinitesimal strain approach, details on the derivation of Eq. (14), a brief summary of our DFT calculations, and an exposition of our generalization of the formula of Slonczewski and Thomas (Ref. [33]) to the nonlinear regime.

[3] M. A. Carpenter, R. J. Hemley, and H.-k. Mao, High-Pressure Elasticity of Stishovite and the $P 42 / \mathrm{mnm} \leftrightarrow \mathrm{Pnnm}$ Phase Transition, J. Geophys. Res. [Solid Earth] 105, 10807 (2000).

[4] M. Guennou, P. Bouvier, G. Garbarino, J. Kreisel, and E. K. H. Salje, Pressure-Induced Phase Transition(s) in
$\mathrm{KMnF}_{3}$ and the Importance of the Excess Volume for Phase Transitions in Perovskite Structures, J. Phys. Condens. Matter 23, 485901 (2011).

[5] P. Bouvier and J. Kreisel, Pressure-Induced Phase Transition in $\mathrm{LaAlO}_{3}$, J. Phys. Condens. Matter 14, 3981 (2002).

[6] D. Errandonea, Landau Theory Applied to Phase Transitions in Calcium Orthotungstate and Isostructural Compounds, Europhys. Lett. 77, 56001 (2007).

[7] A. Togo, F. Oba, and I. Tanaka, First-Principles Calculations of the Ferroelastic Transition between Rutile-Type and $\mathrm{CaCl}_{2}$-Type $\mathrm{SiO}_{2}$ at High Pressures, Phys. Rev. B 78, 134106 (2008).

[8] J. Koppensteiner, A. Tröster, and W. Schranz, Efficient Parametrization of High-Pressure Elasticity, Phys. Rev. B 74, 014111 (2006).

[9] D. C. Wallace, Thermoelasticity of Stressed Materials and Comparison of Various Elastic Constants, Phys. Rev. 162, 776 (1967).

[10] D. C. Wallace, Thermoelastic Theory of Stressed Crystals and Higher-Order Elastic Constants (Academic, Waltham, MA, 1970), p. 301-404.

[11] M. H. Müser and P. Schöffel, Comment on "High-Pressure Elasticity of $\alpha$-Quartz: Instability and Ferroelastic Transition”, Phys. Rev. Lett. 90, 079701 (2003).

[12] L. D. Landau and E. M. Lifshitz, Theory of Elasticity, Course of Theoretical Physics, Vol. 7 (Pergamon, London, 1959).

[13] V. I. Levitas, V. A. Levin, K. M. Zingerman, and E. I. Freiman, Displacive Phase Transitions at Large Strains: Phase-Field Theory and Simulations, Phys. Rev. Lett. 103, 025702 (2009).

[14] V. I. Levitas, A. M. Roy, and D. L. Preston, Multiple Twinning and Variant-Variant Transformations in Martensite: Phase-Field Approach, Phys. Rev. B 88, 054113 (2013).

[15] C. Denoual, A. M. Caucci, L. Soulard, and Y. P. Pellegrini, Phase-Field Reaction-Pathway Kinetics of Martensitic Transformations in a Model $\mathrm{Fe}_{3} \mathrm{Ni}$ Alloy, Phys. Rev. Lett. 105, 035703 (2010).

[16] A. Tröster, W. Schranz, and R. Miletich, How to Couple Landau Theory to an Equation of State, Phys. Rev. Lett. 88, 055503 (2002).

[17] D.C. Wallace, Thermodynamics of Crystals (Dover, New York, 1998).

[18] J. W. Morris and C. R. Krenn, The Internal Stability of an Elastic Solid, Philos. Mag. A 80, 2827 (2000).

[19] M. Born, Thermodynamics of Crystals and Melting, J. Chem. Phys. 7, 591 (1939).

[20] J. Wang, J. Li, S. Yip, S. Phillpot, and D. Wolf, Mechanical Instabilities of Homogeneous Crystals, Phys. Rev. B 52, 12627 (1995).

[21] Z. Zhou and B. Joós, Stability Criteria for Homogeneously Stressed Materials and the Calculation of Elastic Constants, Phys. Rev. B 54, 3841 (1996).

[22] H. Wang and M. Li, Unifying the Criteria of Elastic Stability of Solids, J. Phys. Condens. Matter 24, 245402 (2012).

[23] M. Guennou, P. Bouvier, J. Kreisel, and D. Machon, Pressure-Temperature Phase Diagram of $\mathrm{SrTiO}_{3}$ Up to 53 GPa, Phys. Rev. B 81, 054115 (2010).

[24] R. A. Cowley, The Phase Transition of Strontium Titanate, Phil. Trans. R. Soc. A 354, 2799 (1996). 
[25] Properties and Applications of Perovskite-Type Oxides, edited by L.G. Tejuca and J. L.G. Fierro, Chemical Industries Vol. 50 (Dekker, New York, 1992).

[26] M. Murakami, Y. Ohishi, N. Hirao, and K. Hirose, A Perovskitic Lower Mantle Inferred from High-Pressure, High-Temperature Sound Velocity Data, Nature (London) 485, 90 (2012).

[27] Note that the tensorial coupling parameters $d_{11}^{(2,1)}$ and $d_{33}^{(2,1)}$ used in our generic parametrization scheme differ from those used in Ref. [23], whose coupling parameters $\lambda_{a}^{0}=$ $\left(2 d_{11}^{(2,1)}+d_{33}^{(2,1)}\right) / 3$ and $\lambda_{t}^{0}=\left(d_{33}^{0}-d_{11}^{0}\right) / 2 \sqrt{3}$ are symmetry adapted to the specific transition cubic $\rightarrow$ tetragonal.

[28] P. Blaha, K. Schwarz, G. K. H. Madsen, D. Kvasnicka, and J. Luitz, WIEN2K: An Augmented Plane Wave and Local Orbitals Program for Calculating Crystal Properties (Vienna University of Technology, Vienna, Austria, 2001).

[29] J. P. Perdew, K. Burke, and M. Ernzerhof, Generalized Gradient Approximation Made Simple, Phys. Rev. Lett. 77, 3865 (1996).

[30] J. P. Perdew, K. Burke, and M. Ernzerhof, Generalized Gradient Approximation Made Simple, Phys. Rev. Lett. 77,
3865 (1996); Generalized Gradient Approximation Made Simple, 78, 1396 (1997).

[31] Z. Wu and R. E. Cohen, More Accurate Generalized Gradient Approximation for Solids, Phys. Rev. B 73, 235116 (2006).

[32] J. P. Perdew, A. Ruzsinszky, G. I. Csonka, O. A. Vydrov, G. E. Scuseria, L. A. Constantin, X. Zhou, and K. Burke, Restoring the Density-Gradient Expansion for Exchange in Solids and Surfaces, Phys. Rev. Lett. 100, 136406 (2008).

[33] J. C. Slonczewski and H. Thomas, Interaction of Elastic Strain with the Structural Transition of Strontium Titanate, Phys. Rev. B 1, 3599 (1970).

[34] W. Schranz, A. Tröster, J. Koppensteiner, and R. Miletich, Finite Strain Landau Theory of High Pressure Phase Transformations, J. Phys. Condens. Matter 19, 275202 (2007).

[35] A. V. Kityk, W. Schranz, P. Sondergeld, D. Havlik, E. K. H. Salje, and J. F. Scott, Low-Frequency Superelasticity and Nonlinear Elastic Behavior of $\mathrm{SrTiO}_{3}$ Crystals, Phys. Rev. B 61, 946 (2000). 